\title{
Augmented mitochondrial cytochrome c oxidase activity in children with iron deficiency: a tandem between iron and copper?
}

\author{
Aysenur Bahadir ${ }^{1}$, Erol Erduran ${ }^{1}$, Orhan Değer ${ }^{2}$, Yelda Birinci ${ }^{3}$, Ahmet Ayar ${ }^{4}$
}

\author{
${ }^{1}$ Division of Pediatric Hematology-Oncology, Faculty of Medicine, Karadeniz Technical \\ University, Trabzon, Turkey \\ 2Department of Biochemistry, Faculty of Medicine, Karadeniz Technical University, \\ Trabzon, Turkey \\ ${ }^{3}$ Department of Biochemistry, Sabanci University, Istanbul, Turkey \\ ${ }^{4}$ Department of Physiology, Faculty of Medicine, Karadeniz Technical University, \\ Trabzon, Turkey
}

Submitted: 19 January 2016

Accepted: 21 March 2016

Arch Med Sci 2018; 14, 1: 151-156

DOI: 10.5114 /aoms.2016.59602

Copyright $\odot 2016$ Termedia \& Banach

\section{Abstract}

Introduction: Dyshomeostasis of essential trace elements including iron and copper plays a key role in the pathogenesis of a myriad of serious conditions including iron deficiency (ID) anemia, in which impaired cellular energy metabolism is prominent. Although experimental studies documented decreased activity of cytochrome c oxidase (CytOx) in ID, there are not enough clinical data. The present study was conducted to determine serum copper levels and activity of mitochondrial CytOx in isolated lymphocytes of patients with iron deficiency.

Material and methods: A total of 210 cases (2-17 years) were included in this prospective study. Serum iron and copper levels were measured. According to the serum iron levels, patients were allocated to iron deficient (ID, $n=70)$ and iron deficiency anemia (IDA, $n=70$ ) groups, and iron-sufficient participants were allocated to the control group $(n=70)$. Activity of CytOx in the circulating lymphocytes was colorimetrically measured and compared with the controls. Results: The CytOx activity was significantly higher in the IDA $(2.9 \pm 1.2$ $\mathrm{mOD} / \mathrm{min}, n=62)$ group compared to the control group $(2.4 \pm 1.3 \mathrm{mOD} /$ $\min , n=68, p<0.001)$. Interestingly, serum copper levels were significantly higher in both the ID $(106.9 \pm 55.5 \mu \mathrm{g} / \mathrm{dl}, n=64, p=0.0001)$ and IDA (115.1 $\pm 50.2 \mu \mathrm{g} / \mathrm{dl}, n=59, p=0.0001)$ groups than the control group $(72.1 \pm 46.7$ $\mu \mathrm{g} / \mathrm{dl}, n=69)$.

Conclusions: Higher serum copper levels in patients with IDA implicate co-operative interaction between these trace elements. The elevated CytOx activity in patients with IDA is probably secondary to the normal/elevated serum copper levels.

Key words: iron deficiency, cytochrome c oxidase, mitochondria, copper.

\section{Introduction}

Iron and copper are essential elements, and their deficiencies lead to a myriad of serious conditions [1-4]. Both of these essential trace elements are actively absorbed from the proximal gastrointestinal tract in a regulated manner. When the body needs iron, it is absorbed through di-

\author{
Corresponding author: \\ Dr. Aysenur Bahadir MD \\ Division of Pediatric \\ Hematology-Oncology \\ Faculty of Medicine \\ Karadeniz Technical \\ University \\ TR61080-Trabzon, Turkey \\ Phone: +904623775776 \\ Fax: +904623250518 \\ E-mail: aysenurkbr@ \\ yahoo.com
}


valent metal transporter 1 (DMT1) and transported to the basolateral membrane of enterocytes, and from there into the plasma via ferroportin $[2,5]$.

Of dietary copper, about 30 percent is absorbed through the gastrointestinal tract mucosa and stored in the liver [5-7]. The copper transporter 1 (CTR1) and DMT1 are involved in the absorption of copper from the bowel. Divalent metal transporter 1 in the brush-border membrane has been identified as transporting iron, cadmium, manganese and copper. Previous studies have indicated that the structural disruption of DMT1 affects its expression, and thereby disrupts the transportation of iron and copper primarily [8, 9].

Iron is another essential mineral which is required for several fundamental cell functions, and chronic iron deficiency will result in a myriad of serious conditions. Iron deficiency (ID) and iron deficiency anemia (IDA) affect a significant portion of the world's population [10]. Iron deficiency anemia causes abnormalities in mitochondrial functions in skeletal and cardiac muscles, and also causes impairment in functions of liver and blood cells. It has also been identified that ID leads to decreased cytochrome concentrations in the mitochondria and associated disruption in the respiratory chain [11-14]. Copper is an essential component of the cytochrome $c$ oxidase (CytOx) enzyme, a mitochondrial transmembrane protein. The CytOx enzyme is involved in the final step of the electron transport chain, and is an essential enzyme for oxidative ATP synthesis, with a reaction that also requires iron $[8,15,16]$. CytOx is also involved in oxidative damage and associated programmed cell death [17].

The impact of IDA on cellular energy production capacity is well documented. In experimental animals with IDA, this is further evaluated on enzymes involved in energy production including CytOx, and controversial results are obtained in the neuronal system [18].

Dyshomeostasis of essential trace elements including iron and copper plays a key role in the pathogenesis of a myriad of serious conditions including IDA, in which impaired cellular energy metabolism is prominent. Metalloproteinases, which are regulated by iron and copper, are important cellular components playing key roles in cellular respiration, oxygen transport and cell proliferation. Cytochrome c oxidase contains a heme pocket, which serves as a binding site for copper and iron. It has been documented that there is an interaction between iron and copper in health and disease in all organs and cell types within the organism [19]. However, most of the information is obtained from experimental animal studies [11, $12,18]$, and there are only a few reports on the simultaneous analysis of copper and iron status in humans [4]. Yet iron and copper interdependence and how these interactions relate to health and various disease states are largely unexplained.

The present study was conducted to analyze activity of mitochondrial CytOx in isolated lymphocytes of patients with iron deficiency in relation to serum iron and copper levels.

\section{Material and methods}

This clinical study was conducted at the Division of Pediatric Hematology-Oncology, Karadeniz Technical University Faculty of Medicine. The protocol of the study was approved by the local Ethics Committee and informed consent was obtained from legal representatives of each child before enrollment.

\section{Selection of groups}

Patients admitted to our outpatient clinic between the ages of 2 and 17 years were considered. Complete blood count, serum iron, iron binding capacity and ferritin levels were measured. Inclusion criteria included lower iron levels (ID and IDA), without iron replacement therapy for at least the previous three months and without a history of acute or chronic disorders that may affect the iron metabolism. C-reactive protein (CRP) levels were evaluated to ensure that any high ferritin levels were not associated with inflammation or infection. A CRP value < 0.5 was considered normal.

According to the serum iron levels, the patients were allocated to the following groups:

- Iron deficiency group (ID group): patients who had hemoglobin values within the normal percentile range, ferritin levels above $15 \mathrm{ng} / \mathrm{ml}$ and/or TS below $15 \%$ were considered to have ID.

- Iron deficiency anemia group (IDA group): patients who had hemoglobin values lower than the lowest percentile, ferritin levels below $15 \mathrm{ng} / \mathrm{ml}$ and/or TS below 15\% were considered to have IDA [20, 21].

\section{Control group}

Age and gender matched children who had no acute or chronic disorders that may affect iron metabolism, and who had hemoglobin values within the normal percentile range, ferritin levels $>15 \mathrm{ng} / \mathrm{ml}$, and transferrin saturation (TS) > 15\% were selected as controls.

Thus, a total of 210 cases (70 patients in each group) were included. Only one blood sample was obtained from each patient, from which copper levels and catalase (CAT) and CytOx enzyme activity levels were evaluated. Serum copper levels were analyzed using a spectrometer (Perkin Elmer AAnalyst 800 High Performance Atomic Absorp- 
tion Spectrometer, Perkin-Elmer Corp., MA, USA). The results are expressed as $\mu \mathrm{g} / \mathrm{dl}$.

\section{Lymphocyte isolation}

The lymphocyte isolation in the peripheral blood samples was carried out using Biocoll separating solution (density $1.077 \mathrm{~g} / \mathrm{ml}$, Biological Industries Kibbutz Beit Haemek Israel) based on the principle of density gradient. Blood samples were obtained for the lymphocyte isolation from all of the considered cases; however, the CytOx levels could not be measured in some cases ( 2 out of 70 cases in the control group and 8 out of 70 cases in the IDA group) due to insufficient serum samples or because of hemolysis of the blood sample.

After isolation, the lymphocytes were stored at $-80^{\circ} \mathrm{C}$ in cell culture medium (RPMI medium, Biological Industries, Kibbutz Beit Haemek, Israel) and $10 \%$ dimethyl sulfoxide (DMSO, Sigma, Deisenhofen, Germany) until the day of mitochondria isolation.

\section{Isolation of mitochondria}

Isolation of mitochondria requires the presence of at least $2 \times 10^{8}$ lymphocytes. A blood sample of about $40 \mathrm{ml}$ is required from each case in order to achieve a lymphocyte count of $2 \times 10^{8}$, necessary for the isolation of sufficient mitochondria. In the present study, a maximum of 8-10 $\mathrm{ml}$ of blood was obtained from each child, and for this reason, cases with comparable hemoglobin values from the same group and gender were grouped together to include a minimum of three and a maximum of 8 cases in each subgroup in order to achieve the minimum lymphocyte count of $2 \times 10^{8}$ that is technically required for the isolation of a sufficient amount of mitochondria. Consequently, mitochondria isolation was performed separately in each group of patients and controls, pooled from the lymphocytes isolated from the total of 200 patients. Thus, analysis was performed in a cluster of 13 for the control and ID, while 11 clusters were formed in the IDA group. Despite this clustering, the mean data regarding CytOx and CAT analysis are expressed for the total number of cases in each group.

The isolation of lymphocyte mitochondria was performed using a mitochondria isolation kit (ABCAM, USA), and protein concentrations of the mitochondria were measured using the Lowry procedure [22]. The cells were suspended in Reagent A, containing $5 \mathrm{mg} / \mathrm{ml}$ of protein in the isolation kit, and incubated on ice for $10 \mathrm{~min}$. After freezing, the suspension was homogenized using a homogenizer and the supernatant was stored after centrifugation at $1000 \mathrm{~g}$ for $10 \mathrm{~min}$ at $+4^{\circ} \mathrm{C}$. The remaining pellet was suspended with the Reagent
$B$ provided with the isolation kit to contain $5 \mathrm{mg} /$ $\mathrm{ml}$ protein and incubated on ice for $10 \mathrm{~min}$. After freezing, the suspension was homogenized using a homogenizer and the supernatant was stored after centrifugation at $1000 \mathrm{~g}$ for $10 \mathrm{~min}$ at $+4^{\circ} \mathrm{C}$. The supernatants were mixed thoroughly and the pellets were centrifuged at $12000 \mathrm{~g}$ for $10 \mathrm{~min}$ at $+4^{\circ} \mathrm{C}$. The collected pellet was resuspended in Reagent C, containing a protease inhibitor cocktail (PI) (Sigma, Deisenhofen, Germany) and stored at $-80^{\circ} \mathrm{C}$ until the day of analysis [23].

\section{Lowry protein analysis}

The protein analysis of the samples was performed using the Lowry method, which is based on the reduction of copper ions $\left(\mathrm{Cu}^{+2}\right)$ to $\mathrm{Cu}^{+1}$ through the formation of complex structures with peptide bonds in the proteins in an alkali environment, and the reduction of Folin's phenol reagent by reduced copper ions, and tyrosine, tryptophan and cysteine amino acids in the side chains of the proteins. This causes a colorful reaction that is spectrophotometrically measured at $750 \mathrm{~nm}$ [22].

\section{Analysis of CytOx enzyme activity}

The analysis of activity of the mitochondrial respiratory chain complex IV (also called cytochrome c oxidase, (ytOx) was performed using the Complex IV Human Enzyme Activity Microplate Assay Kit (ABCAM, USA). Protein concentrations for the activity analysis were adjusted according to the ranges recommended for mitochondrial isolates in the kit, and the activity was read at $550 \mathrm{~nm}$ using the Molecular Devices and a Versamax Microplate Reader Spectrophotometer (Sunnyvale, (A, USA).

The results for the enzyme activity were expressed as milliunits per minute (mOD/min). CytOx enzyme activity was measured in 200 cases in which the mitochondria were successfully isolated.

The copper level of some subjects (1 control, 6 ID and 11 IDA) could not be measured due to technical (e.g. sampling, processing) reasons, and the group analysis was performed using the patients with valid copper data.

\section{Analysis of CAT enzyme activity}

CAT enzyme activity was measured using a Mitosciences, Catalase Specific Activity Assay Kit (ABCAM, Istanbul, Turkey). The activity was measured using the luminescence method in a multimode microplate reader (Molecular Devices SpectraMax, USA) according to the kit protocol. Protein concentrations for the activity analysis were adjusted according to the ranges recommended for the protein concentrations of the kit, and the re- 
sults were given according to the Vmax values in the enzyme activity graph. Enzyme activity could not be measured in six cases in the ID group due to technical reasons.

\section{Statistical analysis}

The statistical analysis was performed using the SPSS 13 (Statistical Package for Social Sciences) for Windows software package. The ANOVA test was used for comparison of three groups for normally distributed parameters, and the Mann-Whitney $U$ test was used for non-normally distributed parameters. All results were expressed as mean \pm standard deviation (mean $\pm \mathrm{SD}$ ), and a $p$ value $\leq 0.05$ was considered statistically significant.

\section{Results}

The mean age of the controls, patients with ID and patients with IDA were $10.2 \pm 4.4$ years (range: $2-16,35$ girls and 35 boys), $8.7 \pm 5.1$ years (range: 2-17, 33 girls and 37 boys) and $8.2 \pm 5.8$ years (range: 2-17, 33 girls and 37 boys), respectively. The groups were well matched in terms of age and gender distributions.

The laboratory data of the groups are presented in Table I. The copper levels and CytOx enzyme activities of the groups are presented in Table II.
There was a significant difference between the three groups in terms of serum copper levels $(p=0.0001)$. Copper levels in the ID $(106.9 \pm 55.5$ $\mu \mathrm{g} / \mathrm{dl}, n=64)$ and IDA (115.1 $\pm 50.2 \mu \mathrm{g} / \mathrm{dl}, n=59)$ groups were significantly higher when compared to the control group ( $p=0.0001$ vs. control, for both groups, Table II). The highest serum copper levels were determined in the IDA group; serum copper levels of ID and IDA groups were not significantly different ( $p=0.77$, Table II).

The mean CytOx activity in patients with ID (2.0 $\pm 0.9 \mathrm{mOD} / \mathrm{min}, n=70)$ and patients with IDA (2.9 $\pm 1.2 \mathrm{mOD} / \mathrm{min}, n=62$ ) was not significantly different. The mean CytOx activity of the ID group was lower (but not significantly) than the control ( $2.0 \pm 0.9 \mathrm{mOD} / \mathrm{min}, n=70$ vs. $2.4 \pm 1.3 \mathrm{mOD} / \mathrm{min}$ $n=68, p=0.25)$. The mean CytOx activity of the IDA group was significantly higher in the IDA group than the control $(2.9 \pm 1.2 \mathrm{mOD} / \mathrm{min}, n=62$ vs. 2.4 $\pm 1.3 \mathrm{mOD} / \mathrm{min}, n=68, p<0.001)$. The levels of copper and CytOx activity and statistical comparison of the groups are presented in Table II.

A correlation-regression analysis was carried out to reveal the possible association between mitochondrial CytOx and CAT enzyme activity in the ID and IDA groups. A negative correlation was determined between the mitochondrial CytOx and CAT enzyme activities in the IDA group $(r=-0.49$, $p=0.001)$.

Table I. Laboratory data of study groups

\begin{tabular}{|lccc|}
\hline Parameter & $\begin{array}{c}\text { Control } \\
\text { Mean } \pm \text { SD }\end{array}$ & $\begin{array}{c}\text { ID } \\
\text { Mean } \pm \text { SD }\end{array}$ & $\begin{array}{c}\text { IDA } \\
\text { Mean } \pm \text { SD }\end{array}$ \\
\hline $\mathrm{Hb}[\mathrm{g} / \mathrm{dl}]$ & $12.8 \pm 1.1$ & $12.2 \pm 0.7$ & $9.6 \pm 1.5$ \\
\hline Lymphocyte $[/ \mu \mathrm{l}]$ & $2770 \pm 940$ & $3085 \pm 1283$ & $3268 \pm 1342$ \\
\hline Iron $[\mu \mathrm{g} / \mathrm{dl}]$ & $97.9 \pm 33.4$ & $54.8 \pm 23.4$ & $28.4 \pm 16.3$ \\
\hline Ferritin $[\mathrm{ng} / \mathrm{ml}]$ & $43.6 \pm 34.1$ & $10.6 \pm 3.1$ & $6.8 \pm 4.2$ \\
\hline Copper $[\mu \mathrm{g} / \mathrm{dl}]$ & $72.1 \pm 46.7$ & $106.9 \pm 55.5$ & $115.1 \pm 50.2$ \\
\hline COX $[\mathrm{mOD} / \mathrm{min}]$ & $2.4 \pm 1.3$ & $2.0 \pm 0.9$ & $2.9 \pm 1.2$ \\
\hline CAT $(\mathrm{Vmax})$ & $35097 \pm 12598$ & $30269 \pm 12259$ & $31978 \pm 11173$ \\
\hline
\end{tabular}

ID - iron deficiency, IDA - iron deficiency anemia, $\mathrm{Hb}$ - hemoglobin, COX - cytochrome c oxidase, CAT - catalase, $n$ - number of cases, mean $\pm S D-$ mean \pm standard deviation.

Table II. Levels of serum copper, mitochondrial catalase (CAT) and mitochondrial cytochrome c oxidase (CytOx) enzyme activities among study groups

\begin{tabular}{|lccc|}
\hline Group & \multicolumn{3}{c|}{ Results } \\
\cline { 2 - 4 } & Copper $[\mu \mathrm{g} / \mathrm{dl}]$ & CytOx $[\mathrm{mOD} / \mathrm{min}]$ & CAT $[\mathrm{Vmax}]$ \\
\hline Control & $72.1 \pm 46.7$ & $2.4 \pm 1.3$ & $35097 \pm 12598$ \\
\hline ID & $106.9 \pm 55.5$ & $2.0 \pm 0.9$ & $30269 \pm 12259$ \\
\hline IDA & $115.1 \pm 50.2$ & $2.9 \pm 1.2$ & $31978 \pm 11173$ \\
\hline
\end{tabular}

For copper (control $(n=69)$ vs. ID ( $n=64), p=0.001$; control vs. IDA $(n=59), p=0.001 ;$ ID vs. IDA, $p=0.770)$. For CytOx (control ( $n=68)$ vs. ID ( $n=70), p=0.250$; control vs. IDA $(n=62), p<0.01$; ID vs. IDA, $p=0.250)$. For CAT (control $(n=68) v s$. ID, $p=0.09 ;$ control vs. IDA, $p=0.09 ;$ ID vs. IDA, $p=0.09$ ). 


\section{Discussion}

In the present study, the activity of CytOx enzyme, as the final step in the mitochondrial respiratory chain, was determined to be increased in patients with IDA. In the ID group, CytOx enzyme activity was minimally suppressed when compared to the control group, although the difference was not significant. The highest CytOx enzyme activity was observed in the IDA group.

It is well established that deficiency of iron can cause impairment in cell respiration and cellular energy metabolism $[1,3,11,24]$. Copper is essential for iron utilization. In a study on an iron-deficient rat model it was reported that the copper-carrying protein basolateral membrane copper ATPaz (ATP7A) gene expression was up-regulated, and DMT1 and duodenal cytochrome $b$ (Dcytb) genes were over-expressed [25].

Interaction between the iron and copper in cellular function has been postulated, but although the interaction between the absorption of these metals has been subject to experimental studies the clinical data are rather scarce. Hence, we investigated the serum copper levels and activity of mitochondrial CytOx in patients with different levels of iron deficiency.

In the present study, the serum copper levels in the ID and IDA groups were significantly higher than in the control group. There was no statistically significant difference between the ID and IDA groups, although increased copper levels were more common in patients with ID and higher copper levels were observed in the IDA group. Although we have no direct evidence, it can be postulated that the increased copper levels in the ID and IDA groups could be due to increased absorption of copper through DMT1, which acts as a transporter of divalent metals [25].

Masini et al. [26] observed decreased levels of mitochondrial cytochrome $\mathrm{c}+\mathrm{c} 1$ and $\mathrm{b}$ in the liver of rats with severe IDA. They observed a significant disturbance in energy metabolism of the liver that was associated with mitochondrial dysfunction in cases of severe IDA. Another study conducted on experimental animals with ID reported minimal suppression in cytochrome c concentration, while a significant reduction in cytochrome oxidase activity and copper-containing cytochrome $a+a 3$ concentration has been noted in the livers and hearts of copper-deficient rats when compared to iron-deficient rats [27]. In the study by Dallman et al. [13], cytochrome oxidase activity in control rats with free access to a normal diet did not differ from the cytochrome oxidase activity in rats with ID; however, the enzyme activity in copper-deficient rats was lower than the control group.

In the present study, enhanced CytOx enzyme activity was considered to be associated with in- creased copper absorption from the gastrointestinal tract in response to ID and IDA. No significant correlation was observed between serum copper levels and CytOx enzyme activity in the IDA group $(r=0.12, p=0.4)$. Our results show that mitochondrial enzymes are affected even in the presence of mild iron deficiency, without necessarily development of IDA. Increased mitochondrial CytOx enzyme activity was observed as a result of increasing copper levels in the environment in relation to the decreased iron levels. Another possibility of enhanced CytOx activity could be due to higher reactive oxygen species production. Indeed, there are studies indicating that cytosolic and mitochondrial antioxidant defense system (such as CuZnSOD, MnSOD, catalase and GSH) expression of copper transporter and copper chaperones strikingly increases in dietary copper and iron deficiency in rat $[4,28]$.

On the other hand, the copper-containing CytOx enzyme is one of the important anti-oxidant enzymes in mitochondria. In cases of increased levels of oxidant substances in the mitochondria surpassing the capacity of CytOx enzyme, other anti-oxidant systems may also be activated to remove superoxide ions $[29,30]$. In the presence of excessive copper in the environment, superoxide is formed with the oxidation of copper, resulting in the production of hydrogen peroxide by the activating Haber-Weiss reaction. Hydrogen peroxide also causes tissue damage [31, 32]. In the present study, a negative but not significant correlation was observed between serum copper and CAT enzyme activity in both the ID and IDA groups $(r=$ $-0.05, p=0.7$ and $r=-0.23, p=0.1$, respectively), and it was concluded that the CAT enzyme was not sufficiently functional due to the low levels of iron in both ID and IDA.

Our study has some limitations. Firstly, the age range of the study population was rather large (between 2 and 17 years). Despite this, the mean ages of the groups were comparable and the number of cases at extreme ages was rather small. Although the tendency to ID and IDA differs between these age groups, there is rather minor difference among this population regarding iron and copper metabolism. The clustering of samples, when analyzing the data regarding CytOx and CAT, due to the obtainable volume of blood samples of the population and technical factors for detection of these parameters, is another limitation (see the methods section) of the study.

In conclusion, the results from this cross-sectional clinical study indicate that patients with ID have higher serum levels of copper. This finding indicates that interactions between these two metals are more likely to be cooperative rather than antagonistic. Our findings also documented that CytOx activity is elevated in patients with IDA 
and the increase in the activity of CytOx is probably secondary to the elevated serum copper levels. The iron-copper interactions and possible implication(s) of increased copper levels in ID remain unanswered. The role(s) of augmented activity of this metalloprotein either to support free radical scavenging capacity or support mitochondrial respiration in these patients is not clear and deserves further studies for clarification.

\section{Conflict of interest}

The authors declare no conflict of interest.

\section{References}

1. Iannotti LL, Tielsch JM, Black MM, Black RE. Iron supplemantation in early childhood: health benefits and risks. Am J Clin Nutr 2006; 84: 1261-76.

2. Arredondo M, Nunez MT. Iron and copper metabolism. Mol Aspects Med 2005; 26: 313-3.

3. Malyszko J, Koc-Zorawska E, Levin-laina N, et al. Iron metabolism in hemodialyzed patients - a story half told? Arch Med Sci 2014; 10: 1117-22.

4. Spinazzi M, Sghirlanzoni A, Salviati L, Angelini C. Impaired copper and iron metabolism in blood cells and muscles of patients affected by copper deficiency myeloneuropathy. Neuropathol Appl Neurobiol 2014; 40: 888-98.

5. Wessling-Resnick M. Iron imports. III. Transfer of iron from the mucosa into circulation. Am J Physiol Gastrointest Liver Physiol 2006; 290: G1-6.

6. Brugnara C, Oski FA, Nathan DG. Diagnostic approach to the anemic patients. In: Nathan and Oski's. Hematology of Infancy and Childhood. $7^{\text {th }}$ ed. Orkin SH, Nathan DG, Ginsburg D, Look AT, Fisher DE, Lux SE (eds). Saunders Elsevier, Philadelphia 2009; 455-66.

7. Collins JF, Klevay LM. Copper. Adv Nutr 2011; 2: 520-2.

8. Danks DM. Copper deficiency in humans. Annu Rev Nutr 1988; 8: 235-57.

9. Yoshida K, Furihata K, Takeda S, et al. A mutation in the ceruloplasmin gene is associated with systemic hemosiderosis in humans. Nat Genet 1995; 9: 267-72.

10. Cook JD. Diagnosis and management of iron-deficiency anaemia. Best Pract Res Clin Haematol 2005; 18: 319-32.

11. Liu H, Wang X. Correlation of iron deposition and change of gliocyte metabolism inthe basal ganglia region evaluated usingmagnetic resonance imaging techniques: an in vivo study. Arch Med Sci 2016; 12: 163-71.

12. de Deungria M, Rao R, Wobken JD, Luciana M, Nelson CA, Georgieff MK. Perinatal iron deficiency decreases cytochrome c oxidase (CytOx) activity in selected regions of neonatal rat brain. Pediatr Res 2000; 48: 169-76.

13. Dallman PR. Cytochrome oxidase repair during treatment of copper deficiency: relation to mitochondrial turnover. J Clin Invest 1967; 46: 1819-27.

14. Kwon HE, Lee JH, Lee YM, Kang HC, Lee JS, Kim HD. Iron deficiency in children with mitochondrial disease. Metab Brain Dis 2010; 25: 185-9.

15. Ferguson-Miller S, Hiser C, Liu J. Gating and regulation of the cytochrome C oxidase proton pump. Biochim Biophys Acta 2012; 1817: 489-94.

16. Dennerlein S, Rehling P. Human mitochondrial COX1 assembly into cytochrome $c$ oxidase at a glance. J Cell Sci 2015; 128: 833-7.
17. Kadenbach B, Arnold S, Lee I, Hüttemann M. The possible role of cytochrome $c$ oxidase in stress-induced apoptosis and degenerative diseases. Biochim Biophys Acta 2004; 1655: 400-8.

18. Siddappa AJ, Rao RB, Wobken JD, Leibold EA, Connor JR, Georgieff MK. Developmental changes in the expression of iron regulatory proteins and iron transport proteins in the perinatal rat brain. Neurosci Res 2002; 68: 761-75.

19. Collins JF, Prohaska JR, Knutson MD. Metabolic crossroads of iron and copper. Nutr Rev 2010; 68: 133-47.

20. Ferrari M, Mistura L, Patterson E, et al. Evaluation of iron status in European adolescents through biochemical iron indicators: the HELENA study. Eur J Clin Nutr 2011; 65: 340-9.

21. Dalman PR, Siimes MA. Percentile curves for hemoglobin and red cell volume in infancy and childhood. J Pediatr 1979; 94: 26-38.

22. Lowry O, Rosebrough NJ, Farr AL, Randall RJ. Protein measurement with the Folin phenol reagent. J Biol Chem 1951; 193: 265-75.

23. McComsey GA, Kang M, Ross AC, et al. Increased mtDNA levels without change in mitochondrial enzymes in peripheral blood mononuclear cells of infants born to HIV-infected mothers on antiretroviral therapy. HIV Clin Trials 2008; 9: 126-36.

24. Yoo JH, Maeng HY, Sun YK, et al. Oxidative status in iron-deficiency anemia. J Clin Lab Anal 2009; 23: 319-23.

25. Ranganathan PN, Lu Y, Jiang L, Kim C, Collins JF. Serum ceruloplasmin protein expression and activity increases in iron-deficient rats and is further enhanced by higher dietary copper intake. Blood 2011; 118: 3146-53.

26. Masini A, Salvioli G, Cremonesi P, Botti B, Gallesi D, Ceccarelli D. Dietary iron deficiency in the rat. I. Abnormalities in energy metabolism of the hepatic tissue. Biochim Biophys Acta 1994; 1188: 46-52.

27. Collins JF, Franck CA, Kowdley KV, Ghishan FK. Identification of differentially expressed genes in response to dietary iron deprivation in rat duodenum. Am J Physiol Gastrointest Liver Physiol 2005; 288: G964-71.

28. Konstantinova SG, Jordanova NG, Russanov EM. Effect of dietary copper and iron restriction on aconitase activity and antioxidant capacity of liver, kidney and heart from growing rats. Acta Physiol Pharmacol Bulg 2000; 25: 33-42.

29. Srinivasan S, Avadhani NG. Cytochrome c oxidase dysfunction in oxidative stress. Free Radic Biol Med 2012; 53: 1252-63.

30. Zhang K, Chen Y, Huang X, et al. Expression and clinical significance of cytochrome $c$ oxidase subunit IV in colorectal cancer patients. Arch Med Sci 2016; 12: 68-77.

31. Halliwell B, Gutteridge JMC. Oxygen toxicity, oxygen radicals, transition metals and disease. Biochem J 1984; 219: 1-14.

32. Karimi P, Khavari-Nejad RA, Niknam V, Ghahremaninejad F, Najafi F. The effects of excess copper on antioxidative enzymes, lipid peroxidation, proline, chlorophyll, and concentration of $\mathrm{Mn}, \mathrm{Fe}$, and $\mathrm{Cu}$ in Astragalus neo-mobayenii. Sci World J 2012; 615: 670-6. 